\title{
INCREASING QUALITY OF ANATOMICAL EDUCATION BY INTRODUCTION OF DIGITAL IMAGING INTO DOCUMENTATION OF TOPOGRAPHIC DISSECTIONS
}

\author{
Dáša Slížová, Otakar Krs, Blanka Pospíšilová
}

Charles University in Prague, Faculty of Medicine in Hradec Králové: Department of Anatomy

\begin{abstract}
Summary: Knowledge of topographic anatomy is essential prerequisite for any physician to be successful in professional career. That is why we pay special attention to topographic dissections completing course of normal human anatomy at our department. Dissection procedure is subjected to relatively rigid rules demanding removal of some superficial structures during preparation of deeper layers. Thus some important structures get lost for further learning and also for final evaluation of students knowledge and manual skills. Therefore we decided to introduce digital imaging into the recording of course of preparation, the topographic relationships and unique variations. Digital acquisition of pictures through still digital camera makes possible to bypass the wet photographic process and provides instant graphic inputs for further computer processing. The image files can be further edited, printed, archived on various media and then may be used for instructional, test, evaluative, and publication purposes. Moreover, own original pictorial material is feasible to be presented at anatomy lectures. Practical results of this new approach, besides higher attractiveness and motivation impact, allow us to conclude that systematic digital acquisition of dissection pictures combined with computer processing fairly increases quality of anatomical education.
\end{abstract}

Key words: Human anatomy; Computer graphics; Teaching

Sound knowledge of topographic anatomy is essential for both surgical and conservative medical disciplines. Therefore we are stressing the role of topographic dissections which are being performed at our department during the second semester of general medicine curriculum (42 teaching periods, 45 minutes each).

Basic rule for students' work during topographic practical classes is to dissect human body carefully layer by layer with only necessary removal of tissues. Nevertheless, some important and interesting structures in superficial layers must be removed to reveal deeper ones. In such way, some results of students' work are partly lost for their forthcoming instruction and final evaluation. Therefore we regard photographic documentation of key moments throughout topographic dissection as very important and helpful in meeting criteria of dissection program. Thus it is also possible to capture all substantial synoptical features and unique details of dissected cadavers. Every human body is peculiar in its pattern of variations and may possess clinically important differences in position and appearance of some organs, course of vessels and nerves, and so forth.

Owing to some disadvantages of contemporary analogue technologies concerning reproducibility and flexibility of photographic processing, we decided to use a digital still camera for acquisition of pictures. Aim of this study was to check the possibilities and verify reported advantages of digital camera in getting pictorial documentation from topographic dissection, including capture of unique findings, for further usage in anatomical education.

The use of digital cameras for documentation is no more rare in clinical disciplines as it was only some years ago $^{(1,2,3)}$. Moreover, this sophisticated photographic technology has been used in anatomic pathology, mostly for microphotography ${ }^{(4)}$. On the contrary, we have not found any paper dealing with systematic usage of digital camera for documentation of topographic dissections as yet.

We have used digital camera Olympus Camedia 3030ZOOM. Pictures were taken mostly from „free hand“ without tripod and special lighting, only with built-in flash. Picture files were downloaded to computer via USB port. Images were further processed and edited on Intergraph PC graphic station in Adobe Photoshop version 5.5, archived on CD-R media, and printed by color laser printer Minolta Color Page Pro Plus and color ink printer Epson Stylus Photo EX.

Our experience with use of digital camera for documentation of topographic dissections shows that taken pictures are excellent inputs for further computer processing and photorealistic printing on various media. This camera is user-friendly device with capability to check quality of 

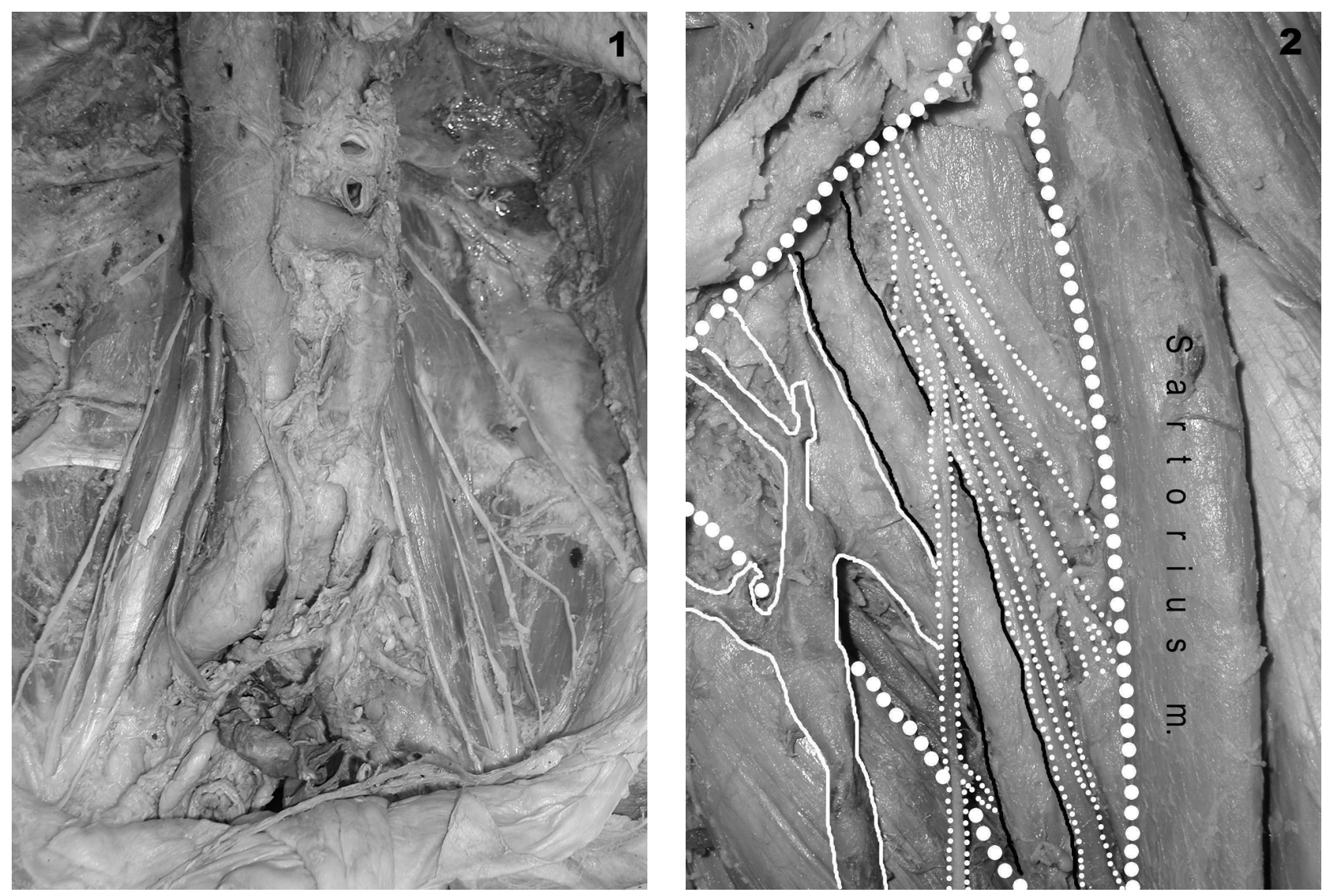

Examples of digital pictorial documentation from topographic dissections and further possibilities of graphic editation for their variable use.

Fig. 1: Retroperitoneal space of abdominal cavity. Overview with dissected large vessels, nerves, and muscles.

Fig. 2: Femoral triangle. Detailed dissection of anterior surface of the thigh. Boundaries of the triangle and the most important structures (greater saphenous vein, femoral artery, vein, and nerve) are highlighted.

pictures directly on camera display. Highly appreciated feasibility to work without demanding lighting and arrangement of photographed objects lets students almost undisturbed during their work. Digital bypassing of classic analogue photographic process enables further direct computer processing without necessity to scan photographs. This results in higher quality and speed of graphics processing and widens availability of pictorial documentation throughout the web.

During the summer semester of the academic year 2000/2001, we were documenting topographic dissection of the entire body. We have taken pictures of opening of the skull and peritoneal cavity and dissection of vertebral canal. Special attention was paid to detailed capturing of consecutive preparation steps of topographically important areas such as the retroperitoneal space (Fig. 1), the limbs (Fig. 2), all layers of the neck (Fig. 3a and 3b), and deep brain structures. These procedures are usually well described and schematically outlined in available resources, how- ever there is usually lack of photorealistic documentation. Printed outputs of selected areas and structures were on hand for instruction before and during practical classes. We have been using digital graphics for current capturing of students' effort and checking results of their work according to dissection program.

Taken photographs can be further edited, printed, archived on various media and used for instructional, test, evaluative, and publication purposes. In spite of increasing amount of information in normal human anatomy available due to development of modern imaging methods, there still remains an exciting way for both students and teachers to take active part in making own original graphic outputs from their everyday work. Regular checking and capturing of dissected areas has also motivating influence on students.

We can conclude that systematic digital acquisition of dissection pictures combined with computer processing fairly increases quality of anatomical education. 

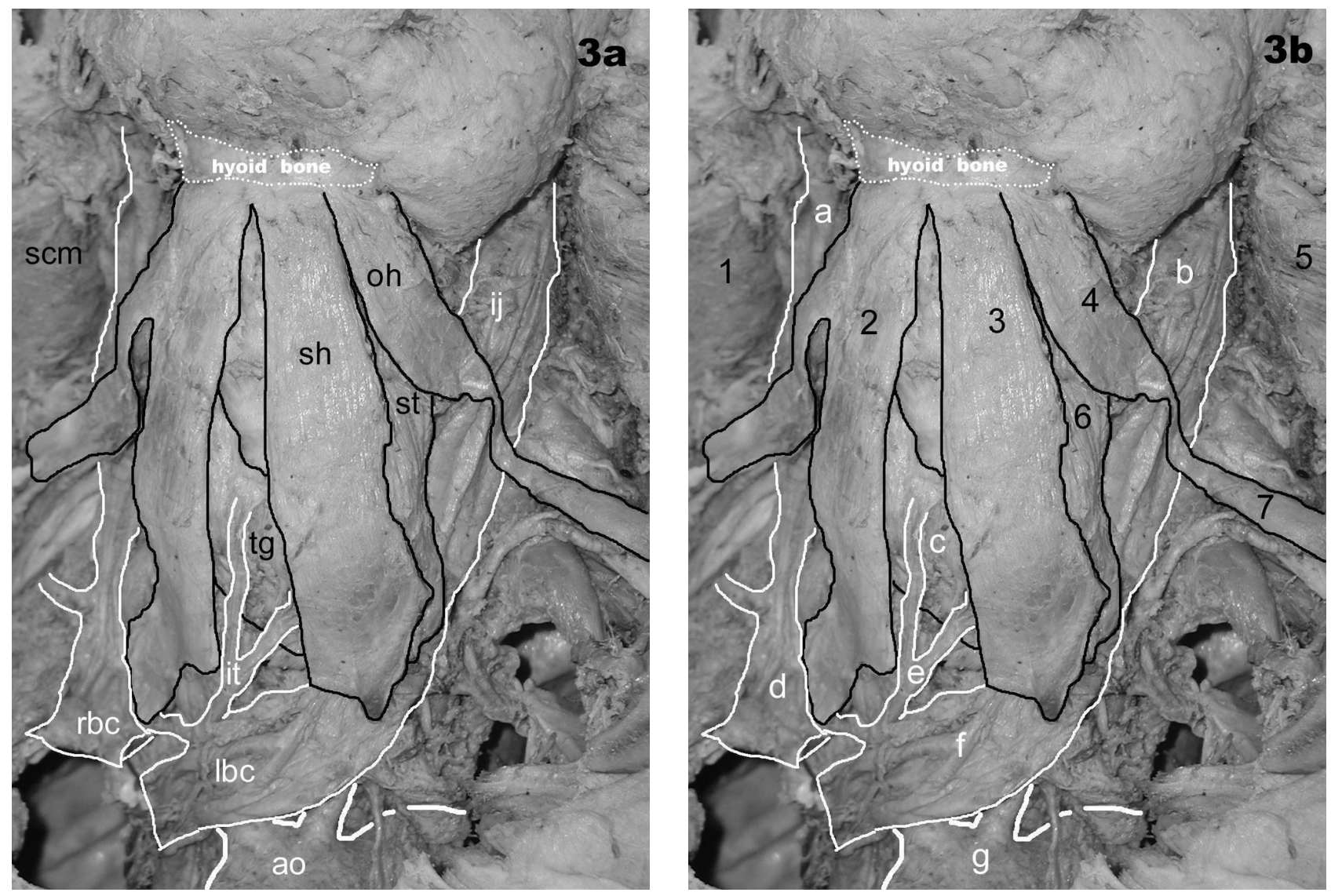

Fig. 3a,b: Anterior region of the neck. The second preparation layer after removal of the platysma muscle. a: Important structures are demarcated for instruction and individual study. The muscles and thyroid gland in black, the vessels in white. b: The same picture adapted for testing. Muscles are numbered and vessels are lettered to distinguish quiz variants for systematic anatomy of muscles or vessels.

\section{References}

1. Sasson M, Schiff T, Stiller MJ. Photography without film: low-cost digital cameras come of age in dermatology. Int J Dermatol 1994;33:113-5.

2. VanderKam VM, Achauer BM. Digital imaging for plastic and reconstructive surgery. Plast Surg Nurs 1997;17:37-8.

3. Elbeshbeshy B, Trepman E. Digital photography in orthopaedic surgery. Foot Ankle Int 2001;22:67-74.

4. Tse CC. Anatomic pathology image capture using a consumer-type digital camera. Am J Surg Pathol 1999;23:155-8.

Submitted November 2001.

Accepted November 2001.

MUDr. Dáša Sližová, CSc., Charles University in Prague, Faculty of Medicine in Hradec Králové, Department of Anatomy, Šimkova 870, 50001 Hradec Králové, Czech Republic. e-mail: slizova@lfhk.cuni.cz 